\title{
Evaluation De La Production De Cinq Genotypes De Palmier A Huile (Elaeis Guinnensis Jacq.) Replantes Sur Des Parcelles A Haute Densite De Fusariose
}

\author{
Désiré Allou \\ Docteur, Chargé de recherche au CNRA; \\ Maitre-Assistant au Laboratoire de génétique \\ Centre National de Recherche Agronomique, Station de Recherche de La \\ Mé, 13 BP 989 Abidjan 13, Côte d'Ivoire. \\ Laboratoire de Génétique, Université Félix Houphouët Boigny, Abidjan- \\ Cocody, UFR des Biosciences, 22 BP 723 Abidjan 22, Côte d'Ivoire.
}

\section{Kouadio Olivier Kouakou}

Master au Laboratoire de génétique

Laboratoire de Génétique, Université Félix Houphouët Boigny, AbidjanCocody, UFR des Biosciences, 22 BP 723 Abidjan 22, Côte d'Ivoire.

\section{Diabaté Sekou}

Docteur, Maitre de Recherche au CNRA, Chef de programme Palmier à huile

Centre National de Recherche Agronomique,

Station de Recherche de La Mé, 13 BP 989 Abidjan 13, Côte d'Ivoire.

\section{Assanvo Simon-Pierre Nguetta}

Professeur titulaire, Directeur du laboratoire de génétique

Laboratoire de Génétique, Université Félix Houphouët Boigny, Abidjan-

Cocody, UFR des Biosciences, 22 BP 723 Abidjan 22, Côte d'Ivoire.

doi: 10.19044/esj.2017.v13n18p562 URL:http://dx.doi.org/10.19044/esj.2017.v13n18p562

\section{Abstract}

Fusarium wilt is the most serious disease of the oil palm (Elaeis guineensis Jacq.) In Africa. The methods of fighting this disease are mainly based on the search for tolerant plant material, so the researchers of CNRA were able to make available to the producers of the genotypes tolerant to the Fusarium wilt. However, two of these supposedly tolerant genotypes, planted on plot D9-3 in Ehania, a highly endemic plot, showed symptoms of vascular Fusarium wilt after 9 years of replanting.

The evaluation of production responses in comparison with the physiological states of other genotypes of the same parental origin showed that the LM25373 and LM25635 progenies retained their ability to produce in spite 
of their doubtful physiological state. The appearance of the external symptoms of FHB did not affect the cycles of production of these so-called diseased progeny.

Keywords: Fusarium disease, Elaeis guineensis, Production, Symptomatic state

\section{Résumé}

La fusariose vasculaire est la maladie la plus grave du palmier à huile (Elaeis guineensis Jacq.) en Afrique. Les méthodes de luttes contre cette maladie sont essentiellement basées sur la recherche de matériel végétal tolérant. C'est ainsi que les chercheurs du CNRA ont pu mettre à la disposition des producteurs des génotypes tolérants à la fusariose. Cependant, deux de ces génotypes censés être tolérants, plantés sur la parcelle D9-3 à Ehania, parcelle hautement endémique, ont présenté des symptômes de la fusariose vasculaire 9 ans après la replantation.

L'évaluation de la production en comparaison des états symptomatiques d'autres génotypes de même origine parentale a montré que les descendances LM25373 et LM25635 ont conservé leur capacité de production en dépit de leur état physiologique douteux. L'apparition de symptômes externes de fusariose n'a pas affectée les cycles de production de ces descendances dites malades.

Mots clés : Fusariose, Elaeis guineensis, Production, Etat symptomatique

\section{Introduction}

Le palmier à huile (Elaeis guineensis Jacq) est une plante d'intérêt économique majeur pour les pays producteurs. Cette plante est actuellement la plante oléagineuse qui a le meilleur rendement en huile à l'hectare. La production mondiale, en 2011 était de 50 millions de tonnes, ce qui représentait $39 \%$ de la production d'huile végétale dont $80 \%$ a été utilisée pour l'alimentation humaine (FAO, 2013). La Côte d'Ivoire, deuxième producteur africain d'huile de palme, a produit 400000 de tonnes d'huile de palme en cette même année (FAO, 2013). Son verger est composé de 215 000 hectares de palmeraie (Hubert, 2010) et couvre la côte sud du pays.

Malheureusement, la culture du palmier à huile est gravement menacée dans cette zone par la fusariose vasculaire, maladie occasionnée par un champignon d'origine tellurique, Fusarium oxysporum f sp. Elaeidis (FOE) (RENARD et de FRANQUEVILLE, 1989). Ce champignon se développe dans les racines puis dans le système vasculaire du stipe. Selon la nature endémique du sol et le matériel végétal utilisé, la fusariose peut causer la destruction de $25 \%$ à $80 \%$ de la palmeraie (DIABATE, 2009). Il n'y a pas 
encore de méthodes de lutte chimiques. C'est pourquoi la recherche sur la fusariose du palmier est basée essentiellement sur la tolérance du matériel végétal à cette maladie (DIABATE, 2013). Des progrès importants ont été réalisés au cours des dix dernières années en matière de sélection de matériel végétal tolérant (GOGBE et al., 2016). Ces résultats sont basés sur les tests d'inoculation de l'agent pathogène au stade deux feuilles (DIABATE et al., 2012) et sur le bon comportement au champ des croisements retenus grâce à un suivi épidémiologique (DIABATE et al., 2015). Les caractéristiques des différentes origines de palmier à huile utilisées dans le programme de sélection pour la tolérance à la fusariose ont été définies (RENARD et al., 1980) en même temps que le caractère polygénique de la résistance, à partir d'un test diallèle. En outre l'hérédité de la tolérance a été identifiée comme étant essentiellement de mode additif. La valeur en test de l'autofécondation des parents peut donc constituer un bon critère de sélection des géniteurs les plus aptes à transmettre leur tolérance (MEUNIER et al., 1979). Le CNRA dispose donc de plusieurs géniteurs tolérants à la fusariose ce qui lui permet de subvenir très largement aux besoins en semences tolérantes en Côte d'ivoire (DURAND-GASSELIN et al., 2000). Cependant, l'apparition des symptômes de la fusariose en 2014 chez les descendances LM25373 et LM25635 plantées sur la parcelle D9-3 à Ehania (Aboisso) en 2005, descendances censées être tolérantes à la fusariose suscite des interrogations même après leurs disparition en 2015; d'où la nécessité d'en évaluer l'effet de cette apparition de la fusariose sur certains caractères de production.

\section{Matériels et méthodes. Matériel végétal.}

Le matériel végétal est composé de 5 descendances d'Elaeis guineensis choisies parmi 20 descendances tolérantes à la fusariose vasculaire, plantées en juillet 2005, après une vieille palmeraie, sur la parcelle D9-3 à EHANIA, zone hautement endémique à la fusariose. La parcelle D9-3, n'a subi aucun traitement chimique ni d'andainage mécanique. La parcelle a été brulée après abattage de la vieille palmeraie. Les descendances choisies sont composées de deux descendances LM25373 et LM25635 qui ont présenté tous deux les symptômes externes et internes de la fusariose vasculaire en 2014 (Figures 1). Leurs cousins descendants respectifs LM25100 et LM25640 des mêmes origines parentales n'ont présenté aucun symptôme de la fusariose vasculaire (Figures 2 et 3). Une descendance témoin LM25286, tolérante, issue du croisement LM2T $\times$ DA115D, dont on connait l'indice de fusariose $(\mathrm{IF}=29)$, a servi de contrôle de production. 


\section{Méthodes.}

\section{Méthode d'évaluation.}

L'objectif de cette évaluation étant de montrer comment l'apparition des symptômes a influencé les paramètres de production, l'hypothèse à vérifier a été que l'apparition des symptômes de la fusariose n'influence pas le cycle de production des descendances censées être tolérantes à cette maladie fongique. C'est-à-dire la production de régimes avant et après l'apparition des symptômes de la fusariose chez les descendances ayant fait la maladie est identique à celle des descendances saines issues de la même origine parentale que ces dernières. Pour évaluer les réponses de production face à l'état physiologiques ou symptomatique des différentes descendances choisies, l'on a procédé à l'analyse de leurs rendements pour chaque variable mesurée année par année de 2013 à 2015.

\section{Dispositif expérimental.}

Le dispositif expérimental originel utilisé pour la plantation était un Lattice carré $5 \times 5$ à 6 répétitions. Ce dispositif avait pour but de tester les 20 descendances. Mais pour l'évaluation de l'incidence de la fusariose sur la production de régimes, les 5 descendances observées ont été choisies en fonction de leur état symptomatique vis-à-vis de la fusariose selon un dispositif en blocs complètement aléatoire. Les dispositions des différentes descendances sur la parcelle sont consignées dans le Tableau I. L'effectif des arbres par descendance est de 96 arbres plantés par lot de 16 en raison de 4 arbres par ligne pour 6 répétitions de parcelles élémentaires.

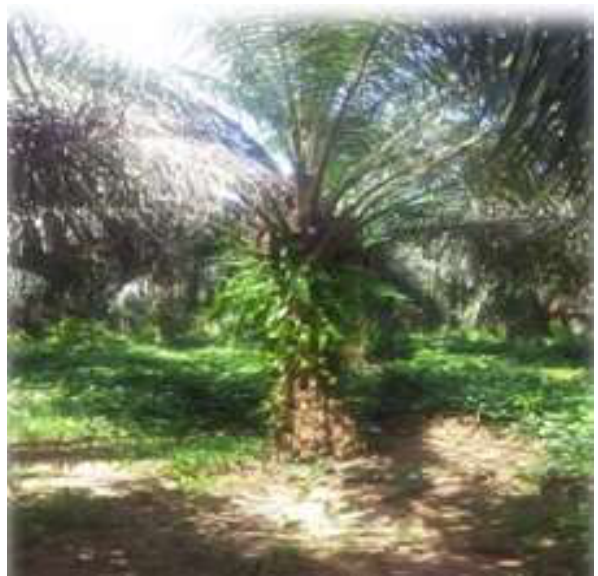

A

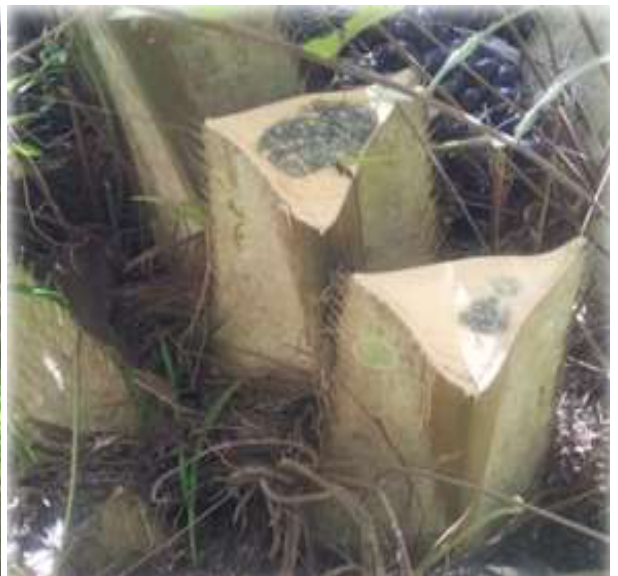

B

Figure 1: Aspect des symptômes externes (A) et internes (B) de la fusariose vasculaire observés sur des plants supposés malades (Photo ALLOU.D, 2014). 


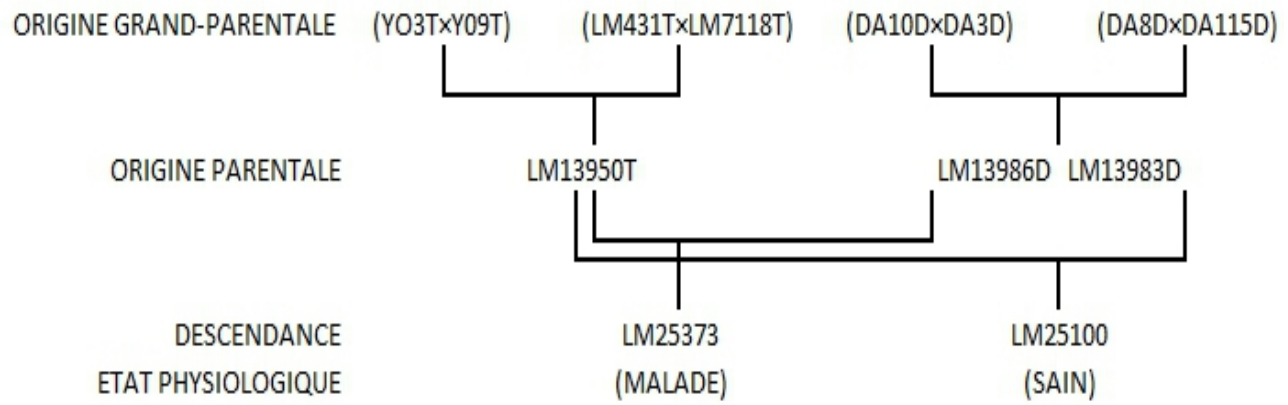

Figure 2: Pedigree des descendances LM25373 et LM25100 issues d'origine parentale commune, tolérant à la fusariose vasculaire.

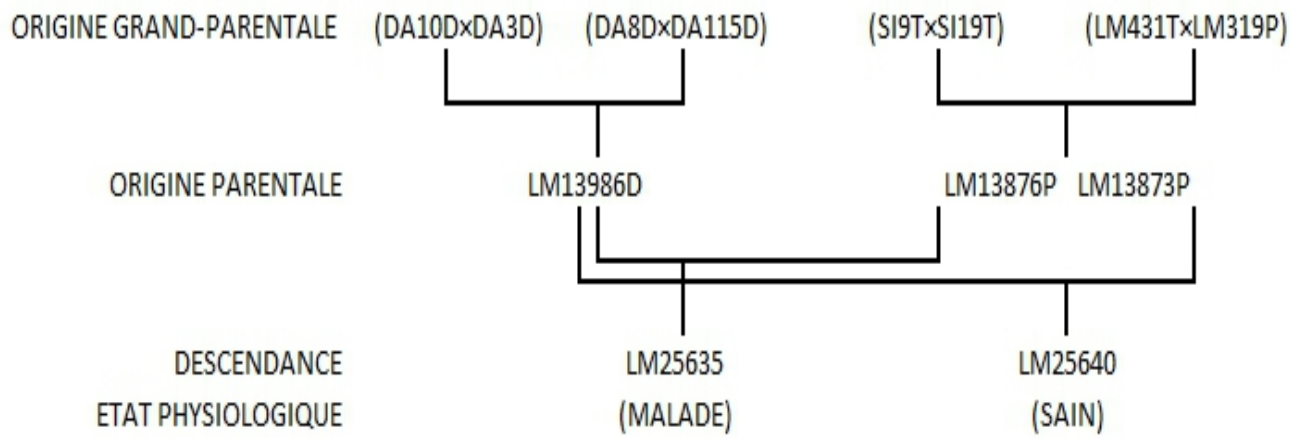

Figure 3: Pedigree des descendances LM25635 et LM25640 issues d'origine parentale commune, tolérante à la fusariose vasculaire.

Tableau I: Disposition des arbres des descendances sur la parcelle D9-3.

\begin{tabular}{|llll|}
\hline PRESENT & $10-13$ & $6-9$ & 16 \\
\hline PRESENT & $30-33$ & $2-5$ & 16 \\
PRESENT & $42-45$ & $22-25$ & 16 \\
PRESENT & $62-65$ & $6-9$ & 16 \\
PRESENT & $82-85$ & $14-17$ & 16 \\
PRESENT & $94-97$ & $14-17$ & 16 \\
\hline ABSENT & $2-5$ & $22-25$ & 16 \\
ABSENT & $30-33$ & $10-13$ & 16 \\
ABSENT & $50-53$ & $14-17$ & 16 \\
ABSENT & $54-57$ & $6-9$ & 16 \\
ABSENT & $66-69$ & $2-5$ & 16 \\
ABSENT & $86-89$ & $22-25$ & 16 \\
\hline PRESENT & $2-5$ & $6-9$ & 16 \\
PRESENT & $22-25$ & $2-5$ & 16 \\
PRESENT & $38-41$ & $10-13$ & 16 \\
PRESENT & $58-61$ & $2-5$ & 16 \\
PRESENT & $82-85$ & $6-9$ & 16 \\
PRESENT & $86-89$ & $14-17$ & 16 \\
\hline ABSENT & $2-5$ & $18-21$ & 16 \\
ABSENT & $18-21$ & $14-17$ & 16 \\
ABSENT & $38-41$ & $14-17$ & 16
\end{tabular}




\begin{tabular}{|llll|}
\hline ABSENT & $66-69$ & $10-13$ & 16 \\
ABSENT & $82-85$ & $2-5$ & 16 \\
ABSENT & $82-85$ & $10-13$ & 16 \\
\hline TEMOIN & $14-17$ & $10-13$ & 16 \\
TEMOIN & $34-37$ & $18-21$ & 16 \\
TEMOIN & $50-53$ & $22-25$ & 16 \\
TEMOIN & $62-65$ & $10-13$ & 16 \\
TEMOIN & $78-81$ & $6-9$ & 16 \\
TEMOIN & $98-101$ & $6-9$ & 16 \\
\hline
\end{tabular}

\section{Variables mesurées.}

$\mathrm{Au}$ stade de maturité du régime, lorsque les premiers fruits mûrs commencent à tomber, le régime est récolté à l'aide de faucille (Figure 4). Le Nombre Total de Régimes (NTR) est déterminé par un dénombrement des régimes récoltés sur chaque arbre par descendance et par mois. Puis un cumul des valeurs mensuelle est détermine par année de production. Une pesée individuelle des régimes récoltés sur chaque arbre par descendance et par mois est aussi réalisée. Le cumul des valeurs mensuelles de pesée permet de calculer la Production Totale de Régimes (PTR), exprimée en Kg. Une estimation du Poids Moyen des Régimes (PMR) sur chaque arbre par descendance et par an est obtenue par la détermination du ratio (PTR/NTR).

Le dénombrement des régimes et la mesure du poids ont été réalisés au pied de chaque arbre, à une fréquence de trois visites de parcelle par mois.

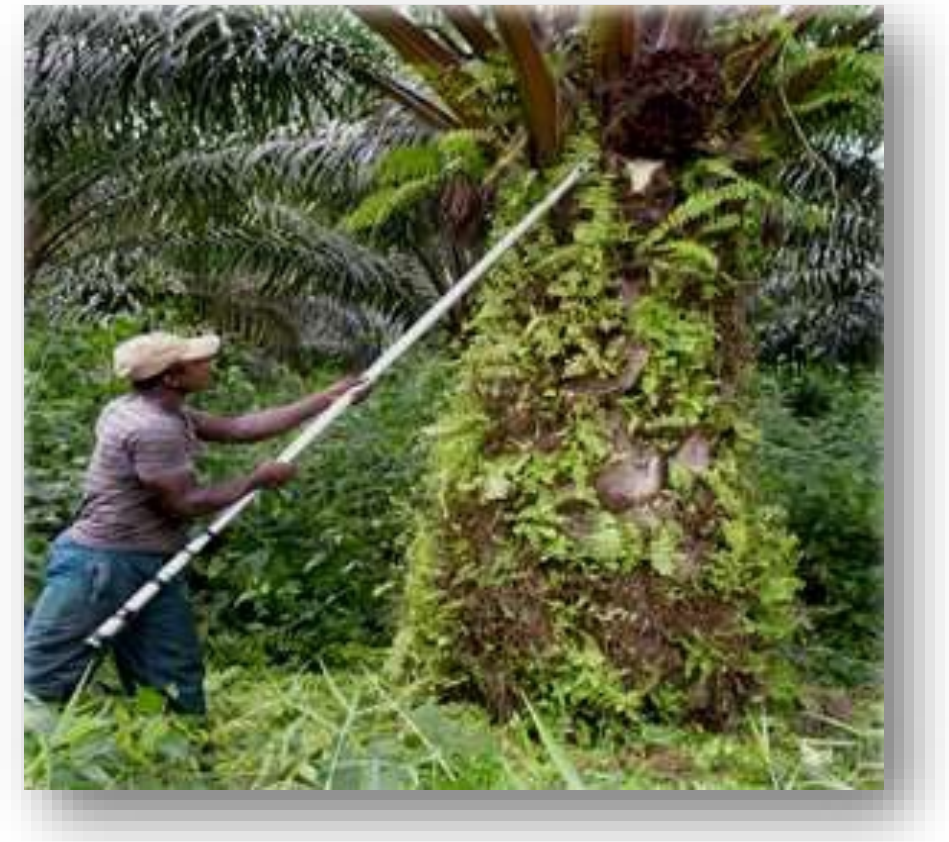

Figure 4: Coupe d'un régime mature à l'aide de faucille (RAFFLEGEAU). 


\section{Analyses statistiques}

Le dispositif expérimental ayant été réaménagé, les valeurs vérifiées, l'hypothèse de normalité rejetée (ROYSTON, 1995) et l'examen d'homogénéité des variances inacceptable (SNEDECOR et COCHRAN, 1980), les tests paramétriques (DAGNELIE, 1986) n'ont donc pas été utilisés pour apprécier les différentes entre cycle de production. Les analyses statistiques utilisées ont été basées sur les tests non paramétriques (SIEGEL, 1995). Le test de KRUSKAL-WALLIS a été utilisé comme une alternative à l'ANOVA. Cette analyse de variances de KRUSKAL-WALLIS a été réalisée pour détecter des différences significatives, au risque de 5\%, en fonction des cycles de production (2013, 2014 et 2015). La formation de groupes homogènes a été effectuée grâce au test de comparaisons multiples des rangs moyens des descendances testées. L'ensemble des tests a été effectué à l'aide du logiciel STATISTICAT 7.1.

\section{Résultats.}

\section{Comparaison du Nombre Total de Régimes, NTR.}

Le Nombre Total de Régimes (NTR) produit par les descendances diffère d'une année à l'autre (Figure 4). En effet, NTR qui était meilleur en 2013 a baissé en 2014, année de l'apparition des symptômes de la fusariose, pour augmenter légèrement en 2015 après la disparition des symptômes. Malgré cette variation dans le cycle de production, les descendances ont conservé leurs rangs les uns par rapport aux autres. Les résultats des tests d'analyses ont montré que pour l'année 2013, il a existé une différence significative entre les moyennes des rangs des descendances. Le classement a révélé que la descendance témoin LM25286 a eu le meilleur rendement. Elle a été suivie par les descendances saines LM25640 et LM25100, puis par les descendances malades LM25373 et LM25635. En 2014, il a aussi existé une différente significative entre les moyennes des rangs des descendances. Le classement est resté identique à celui de l'année 2013. Pour l'année 2015, il a encore existé une différence significative entre les moyennes des rangs des descendances. Le classement est encore resté le même qu'en années précédentes (2013 et 2014) (Tableau II). De façon générale, au sein d'une même origine généalogique, les moyennes des rangs diffèrent d'un état physiologique à l'autre. Cependant, l'existence de l'effet descendance sur le Nombre Total de Régimes (NTR) ne saurait être liée à l'état symptomatique des arbres. En effet, la différence de NTR observée entre LM25373 (malade) et LM25100 (sain) est restée non significative (Tableau III). 


\section{Comparaison du Poids Total de Régimes, PTR.}

Le Poids Total de Régimes (PTR) des descendances diffère d'une année à l'autre (Figure 6). Le PTR de l'année 2013 est meilleur que celui de 2015 qui semble être plus élevé que celui de 2014. Les résultats des tests d'analyse (Tableau IV) ont révélé que le PTR de l'année 2013 (année avant l'apparition des symptômes de la fusariose) diffère significativement d'une descendance à l'autre. Le classement des descendances montre que le témoin (LM25286) a le meilleur PTR.

\section{NTR}

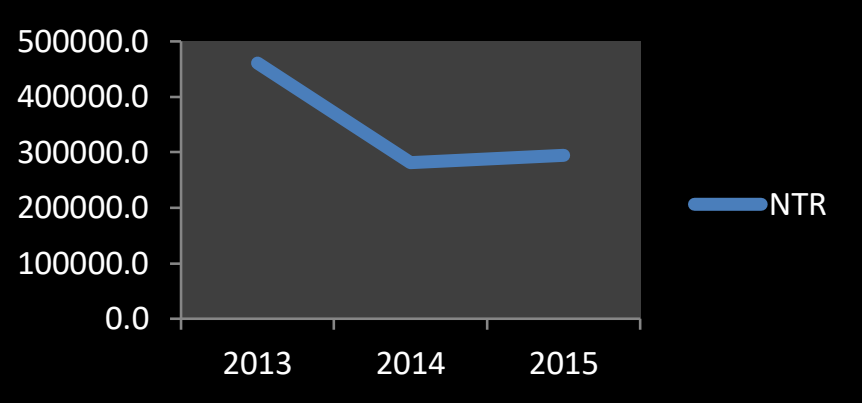

Figure 5: Evolution du NTR en fonction des années de productions.

\section{PTR}

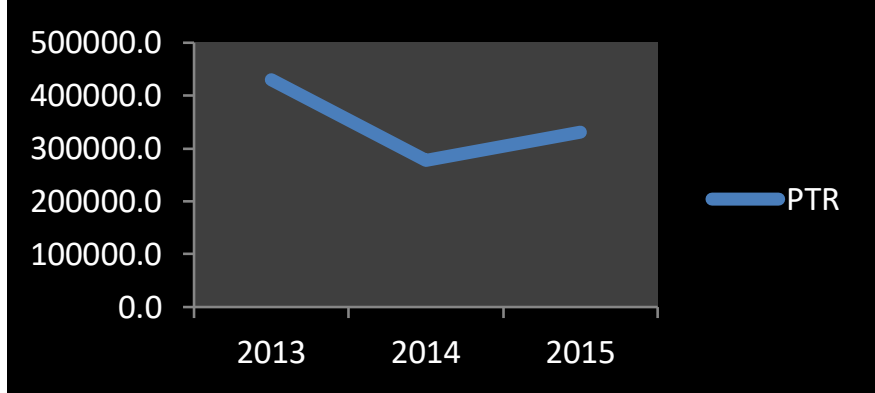

Figure 6: Evolution du PTR en fonction des années de productions

\section{PMR}

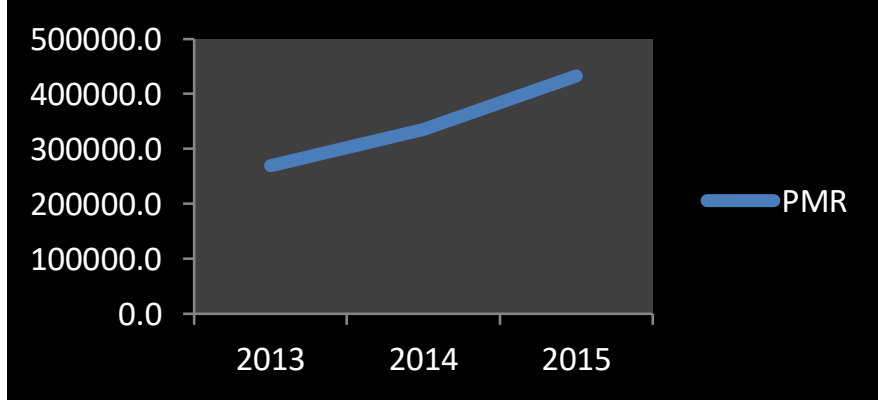

Figure 7: Evolution du PMR en fonction des années de production 
Ce dernier est suivi par les descendances LM25640 (saine), LM25373 (malade), LM25100 (saine) et la descendance LM25635 (malade). En année 2014, il a aussi existé une différence significative entre les moyennes des rangs des descendances. Le classement des descendances a montré que le témoin LM25286 a conservé son premier rang suivi de la descendance LM25100 (saine), puis de la descendance LM25373 (malade) et de la descendance LM25640 (saine). La descendance LM25635 (malade) s'est classée en dernière position (Tableau IV). En année 2015, il a encore existé une différence significative entre les moyennes des rangs des descendances. Le classement a montré que le témoin LM25286 a encore eu le meilleur PTR, il a été suivi par les descendances saines LM25640 et LM25100, puis par les descendances malades LM25373 et LM25635. De façon générale, au sein d'une même origine généalogique, les moyennes des rangs diffèrent d'un état physiologique à l'autre. Cependant, l'existe de l'effet descendance sur le Poids Total de Régimes (PTR) ne pourrait être lié à l'état symptomatique des arbres. En effet, la différence de PTR observée entre LM25373 (malade) et LM25100 (sain) est restée non significative (Tableau V).

\section{Comparaison du Poids Moyen de Régimes, PMR.}

Le Poids Moyen de Régimes (PMR) produit par les descendances diffère d'une année à l'autre (Figure 7). Le PMR qui était faible en 2013 a augmenté en 2014, année de l'apparition des symptômes de la fusariose, pour augmenter plus encore en 2015 après la disparition des symptômes. En année 2013, la différence entre les moyennes des rangs des descendances a été significative. La descendance LM25373 (malade) a eu le meilleur poids moyen. Cette descendance a été suivie par les descendances LM25635 (malade) et LM25100 (saine) puis le témoin et enfin en dernière position par la descendance LM25640 (saine). En année 2014, année de l'apparition des symptômes de la fusariose, il a existé aussi une différente significative entre les moyennes des rangs. La descendance malade LM25373 a conservé sa position de $1^{\text {er }}$ rang du classement de 2013. En année 2015, année de la disparition des symptômes de la fusariose, la différence entre les moyennes des rangs est encore restée significative. Toutes les descendances malades ou saines se sont mieux comportées que le témoin. En général, les descendances se sont mieux comportées malgré leur l'état symptomatique. Trois groupes homogènes ont été formés à l'issu du test de comparaisons multiples des rangs. (Tableau VI et VII).

Tableau II: Classement des descendances en fonction des moyennes de rang du NTR

\begin{tabular}{|c|c|c|c|c|c|c|c|c|}
\hline $\begin{array}{c}\text { DESCEDANC } \\
\text { E }\end{array}$ & 2013 & 2014 & 2015 & $\begin{array}{c}\text { Rang } \\
2013\end{array}$ & $\begin{array}{c}\text { Rang } \\
2014\end{array}$ & $\begin{array}{c}\text { Ran } \\
201 \\
5\end{array}$ & $\begin{array}{c}\text { Grou } \\
\text { pe }\end{array}$ & ETAT \\
\hline
\end{tabular}




\begin{tabular}{|c|c|c|c|c|c|c|c|c|}
\hline LM 25373 & $\begin{array}{c}19601, \\
00\end{array}$ & $\begin{array}{c}21701, \\
00\end{array}$ & $\begin{array}{c}17702, \\
50\end{array}$ & 4 & 4 & 4 & C & $\begin{array}{c}\text { MALA } \\
\text { DE }\end{array}$ \\
\hline LM25100 & $\begin{array}{c}21662, \\
00\end{array}$ & $\begin{array}{c}24130, \\
00\end{array}$ & $\begin{array}{c}23741, \\
50\end{array}$ & 3 & 3 & 3 & B C & SAIN \\
\hline LM 25635 & $\begin{array}{c}13935, \\
00\end{array}$ & $\begin{array}{c}14400, \\
00\end{array}$ & $\begin{array}{c}14088, \\
00\end{array}$ & 5 & 5 & 5 & & $\begin{array}{c}\text { MALA } \\
\text { DE }\end{array}$ \\
\hline LM 25640 & $\begin{array}{c}27707, \\
00\end{array}$ & $\begin{array}{c}24917, \\
00\end{array}$ & $\begin{array}{c}26991, \\
00\end{array}$ & 2 & 2 & 2 & B & SAIN \\
\hline LM 25286 & $\begin{array}{c}32535, \\
00\end{array}$ & $\begin{array}{c}30292, \\
00\end{array}$ & $\begin{array}{c}32917, \\
00\end{array}$ & 1 & 1 & 1 & A & $\begin{array}{c}\text { TEMO } \\
\text { IN }\end{array}$ \\
\hline $\begin{array}{c}\text { Test de kruskal- } \\
\text { wallis } \\
\text { H ( 4, N=480) }\end{array}$ & \begin{tabular}{c}
113,42 \\
\hline
\end{tabular} & $\begin{array}{c}72,91 \\
121,35\end{array}$ & \multicolumn{3}{|c|}{$\mathrm{H}>\chi^{2}$ lu } & \\
\hline
\end{tabular}

Tableau III: Valeur p des comparaisons multiples des descendances en fonction de NTR.

\begin{tabular}{|c|c|c|c|c|c|c|c|}
\hline \multicolumn{3}{|c|}{ Descendance } & \multicolumn{5}{|c|}{$\begin{array}{c}\text { Valeurs p des Comparaisons Multiples (bilatéral) de la } \\
\text { Variable indépendante NTR ; classement des } \\
\text { descendances par le Test de Kruskal-Wallis: H (4, N= } \\
1440)=238,5776 \mathrm{p}=0,001\end{array}$} \\
\hline & $\begin{array}{l}\text { Grands- } \\
\text { parents }\end{array}$ & Etat & $\begin{array}{c}\text { LM253 } \\
73\end{array}$ & $\begin{array}{c}\text { LM251 } \\
00\end{array}$ & $\begin{array}{c}\text { LM256 } \\
35\end{array}$ & $\begin{array}{c}\text { LM256 } \\
40\end{array}$ & $\begin{array}{c}\text { LM252 } \\
86\end{array}$ \\
\hline $\begin{array}{c}\text { LM253 } \\
73\end{array}$ & $\begin{array}{c}(\text { YO3T } \times \text { YO9 } \\
\text { T) }\end{array}$ & $\begin{array}{c}\text { Malad } \\
\text { e }\end{array}$ & & $\begin{array}{c}0,07915 \\
8 \\
\end{array}$ & $\begin{array}{c}0,00001 \\
9\end{array}$ & $\begin{array}{c}0,00000 \\
1\end{array}$ & $\begin{array}{c}0,00000 \\
1\end{array}$ \\
\hline $\begin{array}{c}\text { LM251 } \\
00 \\
\end{array}$ & $\begin{array}{c}\text { (YO3T } \times \text { YO9 } \\
\text { T) }\end{array}$ & Sain & $\begin{array}{c}0,07915 \\
8 \\
\end{array}$ & & $\begin{array}{c}0,00000 \\
1\end{array}$ & $\begin{array}{c}0,06504 \\
1 \\
\end{array}$ & $\begin{array}{c}0,00000 \\
1\end{array}$ \\
\hline $\begin{array}{c}\text { LM256 } \\
35\end{array}$ & $\begin{array}{c}(\text { SI9T } \times \text { SI19T } \\
)\end{array}$ & $\begin{array}{c}\text { Malad } \\
\text { e }\end{array}$ & $\begin{array}{c}0,00001 \\
9\end{array}$ & $\begin{array}{c}0,00000 \\
1\end{array}$ & & $\begin{array}{c}0,00000 \\
1\end{array}$ & $\begin{array}{c}0,00000 \\
1\end{array}$ \\
\hline $\begin{array}{c}\text { LM256 } \\
40\end{array}$ & $\begin{array}{c}(\text { SI9T } \times \text { SI19T } \\
\text { (1) }\end{array}$ & Sain & $\begin{array}{c}0,00000 \\
1\end{array}$ & $\begin{array}{c}0,06504 \\
1\end{array}$ & $\begin{array}{c}0,00000 \\
1\end{array}$ & & $\begin{array}{c}0,00017 \\
4\end{array}$ \\
\hline $\begin{array}{l}\text { LM252 } \\
86\end{array}$ & Témoin & & $\begin{array}{c}0,00000 \\
1\end{array}$ & $\begin{array}{c}0,00000 \\
1\end{array}$ & $\begin{array}{c}0,00000 \\
1\end{array}$ & $\begin{array}{c}0,00017 \\
4\end{array}$ & \\
\hline
\end{tabular}

Les valeurs de $p$ du test en italique indiquent qu'il existe une différence significative entre la ligne et la colonne

Les valeurs de $p$ du test en gras indiquent qu'il n'existe pas de différence significative entre la ligne et la colonne

Tableau IV : Classement des descendances en fonction des moyennes de rang du PTR.

\begin{tabular}{|c|c|c|c|c|c|c|c|c|}
\hline DESCEDANCE & 2013 & 2014 & 2015 & $\begin{array}{l}\text { Rang } \\
2013\end{array}$ & $\begin{array}{l}\text { Rang } \\
2014\end{array}$ & $\begin{array}{c}\text { Ran } \\
\text { g } \\
201 \\
5\end{array}$ & $\begin{array}{l}\text { Gro } \\
\text { upe }\end{array}$ & ETAT \\
\hline LM 25373 & $\begin{array}{c}22654 \\
50\end{array}$ & $\begin{array}{c}24018, \\
00\end{array}$ & $\begin{array}{c}20143, \\
50\end{array}$ & 3 & 3 & 4 & B & $\begin{array}{c}\text { MALA } \\
\text { DE }\end{array}$ \\
\hline LM25100 & $\begin{array}{c}21666, \\
00\end{array}$ & $\begin{array}{c}24050, \\
00\end{array}$ & $\begin{array}{c}24031, \\
00\end{array}$ & 4 & 2 & 3 & $\mathrm{~B}$ & SAIN \\
\hline LM 25635 & $\begin{array}{c}13730, \\
00\end{array}$ & $\begin{array}{c}14640, \\
00\end{array}$ & $\begin{array}{c}13535, \\
00\end{array}$ & 5 & 5 & 5 & $\mathrm{C}$ & $\begin{array}{c}\text { MALA } \\
\text { DE }\end{array}$ \\
\hline LM 25640 & $\begin{array}{c}25836 \\
50\end{array}$ & $\begin{array}{c}23011, \\
50\end{array}$ & $\begin{array}{c}26572, \\
50\end{array}$ & 2 & 4 & 2 & B & SAIN \\
\hline
\end{tabular}




\begin{tabular}{|c|c|c|c|c|c|c|c|c|}
\hline LM 25286 & $\begin{array}{c}31553, \\
00\end{array}$ & $\begin{array}{c}29720, \\
50\end{array}$ & $\begin{array}{c}31158, \\
00\end{array}$ & 1 & 1 & 1 & A & $\begin{array}{c}\text { TEMOI } \\
\mathrm{N}\end{array}$ \\
\hline $\begin{array}{c}\text { Test de kruskal- } \\
\text { wallis } \\
\text { H ( } 4, \mathrm{~N}=480)\end{array}$ & 113,42 & 72,91 & 121,35 & \multicolumn{6}{|c|}{$\mathrm{H}>\chi^{2}$ lu } \\
\hline
\end{tabular}

Tableau V: Valeur p des comparaisons des descendances en fonction de PTR

\begin{tabular}{|c|c|c|c|c|c|c|c|}
\hline \multicolumn{3}{|c|}{ Descendance } & \multicolumn{5}{|c|}{$\begin{array}{l}\text { Valeurs p des Comparaisons Multiples (bilatéral) de la } \\
\text { Variable indépendante PTR ; classement des } \\
\text { descendances par le Test de Kruskal-Wallis: H (4, N= } \\
1440)=210,0500 \mathrm{p}=0,001\end{array}$} \\
\hline & $\begin{array}{l}\text { Grands- } \\
\text { parents }\end{array}$ & Etat & $\begin{array}{c}\text { LM253 } \\
73\end{array}$ & $\begin{array}{c}\text { LM251 } \\
00\end{array}$ & $\begin{array}{c}\text { LM256 } \\
35\end{array}$ & $\begin{array}{c}\text { LM256 } \\
40\end{array}$ & $\begin{array}{c}\text { LM252 } \\
86\end{array}$ \\
\hline $\begin{array}{c}\text { LM253 } \\
73\end{array}$ & $\begin{array}{c}(\text { YO3T } \times \text { YO9 } \\
\text { T) }\end{array}$ & $\begin{array}{c}\text { Malad } \\
\text { e }\end{array}$ & & $\begin{array}{c}1,00000 \\
0\end{array}$ & $\begin{array}{c}0,00000 \\
1\end{array}$ & $\begin{array}{c}0,22823 \\
0\end{array}$ & $\begin{array}{c}0,00000 \\
1\end{array}$ \\
\hline $\begin{array}{c}\text { LM251 } \\
00 \\
\end{array}$ & $\begin{array}{c}\text { (YO3T } \times \text { YO9 } \\
\text { T) } \\
\end{array}$ & Sain & $\begin{array}{c}1,00000 \\
0\end{array}$ & & $\begin{array}{c}0,00000 \\
1\end{array}$ & $\begin{array}{c}0,94222 \\
2 \\
\end{array}$ & $\begin{array}{c}0,00000 \\
1\end{array}$ \\
\hline $\begin{array}{c}\text { LM256 } \\
35\end{array}$ & $\begin{array}{c}\text { SI9T } \times \text { SI19T } \\
\text { (SI) }\end{array}$ & $\begin{array}{c}\text { Malad } \\
\text { e }\end{array}$ & $\begin{array}{c}0,00000 \\
1\end{array}$ & $\begin{array}{c}0,00000 \\
1\end{array}$ & & $\begin{array}{c}0,00000 \\
1\end{array}$ & $\begin{array}{c}0,00000 \\
1\end{array}$ \\
\hline $\begin{array}{c}\text { LM256 } \\
40\end{array}$ & $\begin{array}{c}(\text { SI9T } \times \text { SI19T } \\
)\end{array}$ & Sain & $\begin{array}{c}0,22823 \\
0\end{array}$ & $\begin{array}{c}0,94222 \\
2 \\
\end{array}$ & $\begin{array}{c}0,00000 \\
1\end{array}$ & & $\begin{array}{c}0,00002 \\
1\end{array}$ \\
\hline $\begin{array}{l}\mathrm{LM} 252 \\
86\end{array}$ & Témoin & & $\begin{array}{c}0,00000 \\
1\end{array}$ & $\begin{array}{c}0,00000 \\
1\end{array}$ & $\begin{array}{c}0,00000 \\
1\end{array}$ & $\begin{array}{c}0,00002 \\
1\end{array}$ & \\
\hline
\end{tabular}

Les valeurs de $p$ du test en italique indiquent qu'il existe une différence significative entre la ligne et la colonne

Les valeurs de $p$ du test en gras indiquent qu'il n'existe pas de différence significative entre la ligne et la colonne

Tableau VI: Classification des descendances en fonction des moyennes de rang du PMR.

\begin{tabular}{|c|c|c|c|c|c|c|c|c|}
\hline $\begin{array}{c}\text { DESCEDAN } \\
\text { CE }\end{array}$ & 2013 & 2014 & 2015 & $\begin{array}{c}\text { Ran } \\
\text { g } \\
201 \\
3\end{array}$ & $\begin{array}{c}\text { Ran } \\
\mathrm{g} \\
201 \\
4\end{array}$ & $\begin{array}{c}\text { Ran } \\
\text { g } \\
201 \\
5\end{array}$ & $\begin{array}{c}\text { Group } \\
\mathrm{e}\end{array}$ & ETAT \\
\hline LM25373 & $\begin{array}{c}29532,5 \\
0\end{array}$ & $\begin{array}{c}27914,5 \\
0\end{array}$ & $\begin{array}{c}28948,5 \\
0\end{array}$ & 1 & 1 & 1 & A & $\begin{array}{c}\text { MALAD } \\
\text { E }\end{array}$ \\
\hline LM25100 & $\begin{array}{c}24337,0 \\
0\end{array}$ & $\begin{array}{c}23524,5 \\
0\end{array}$ & $\begin{array}{c}24326,5 \\
0\end{array}$ & 3 & 2 & 2 & B & SAIN \\
\hline LM25635 & $\begin{array}{c}24546,5 \\
0\end{array}$ & $\begin{array}{c}21934,0 \\
0 \\
\end{array}$ & $\begin{array}{c}20351,5 \\
0 \\
\end{array}$ & 2 & 4 & 4 & B C & $\begin{array}{c}\text { MALAD } \\
\text { E }\end{array}$ \\
\hline LM25640 & $\begin{array}{c}17961,0 \\
0 \\
\end{array}$ & $\begin{array}{c}19333,5 \\
0 \\
\end{array}$ & $\begin{array}{c}21884,5 \\
0 \\
\end{array}$ & 5 & 5 & 3 & $\mathrm{C}$ & SAIN \\
\hline LM25286 & $\begin{array}{c}19063,0 \\
0 \\
\end{array}$ & $\begin{array}{c}22733,5 \\
0 \\
\end{array}$ & $\begin{array}{c}19929,0 \\
0 \\
\end{array}$ & 4 & 3 & 5 & $\mathrm{C}$ & $\begin{array}{c}\text { TEMOI } \\
\mathrm{N}\end{array}$ \\
\hline $\begin{array}{c}\text { Test de } \\
\text { kruskal-wallis } \\
\mathrm{H}(4, \mathrm{~N}=480)\end{array}$ & 113,42 & 72,91 & 121,35 & \multicolumn{5}{|c|}{$\mathrm{H}>\chi^{2} \mathrm{lu}$} \\
\hline
\end{tabular}


Tableau VII: Valeur p des comparaisons des descendances en fonction de PMR.

\begin{tabular}{|c|c|c|c|c|c|c|c|}
\hline \multicolumn{3}{|c|}{ Descendance } & \multicolumn{5}{|c|}{$\begin{array}{l}\text { Valeurs p des Comparaisons Multiples (bilatéral) de la } \\
\text { Variable indépendante PMR ; classement des } \\
\text { descendances par le Test de Kruskal-Wallis: H (4, N= } \\
1440)=68,05654 \mathrm{p}=, 0001\end{array}$} \\
\hline & $\begin{array}{l}\text { Grands- } \\
\text { parents }\end{array}$ & Etat & $\begin{array}{c}\text { LM } \\
\mathbf{2 5 3 7 3}\end{array}$ & $\begin{array}{c}\text { LM251 } \\
00\end{array}$ & $\begin{array}{c}\text { LM } \\
25635\end{array}$ & $\begin{array}{c}\text { LM } \\
25640\end{array}$ & $\begin{array}{c}\text { LM } \\
25286\end{array}$ \\
\hline $\begin{array}{c}\text { LM253 } \\
73 \\
\end{array}$ & $\begin{array}{c}(\text { YO3T } \times \text { YO9 } \\
\text { T) } \\
\end{array}$ & $\begin{array}{c}\text { Malad } \\
\mathrm{e}\end{array}$ & & $\begin{array}{c}0,00215 \\
9 \\
\end{array}$ & $\begin{array}{c}0,00000 \\
1 \\
\end{array}$ & $\begin{array}{c}0,00000 \\
1\end{array}$ & $\begin{array}{c}0,00000 \\
1 \\
\end{array}$ \\
\hline $\begin{array}{c}\mathrm{LM} 251 \\
00 \\
\end{array}$ & $\begin{array}{c}\text { YO3T } \times \text { YO9 } \\
\text { T) } \\
\end{array}$ & Sain & $\begin{array}{c}0,00215 \\
9 \\
\end{array}$ & & $\begin{array}{c}1,00000 \\
0\end{array}$ & $\begin{array}{c}0,00222 \\
1 \\
\end{array}$ & $\begin{array}{c}0,04417 \\
9 \\
\end{array}$ \\
\hline $\begin{array}{c}\text { LM256 } \\
35\end{array}$ & $\begin{array}{c}(\text { SI9T } \times \text { SI19T } \\
)\end{array}$ & $\begin{array}{c}\text { Malad } \\
\mathrm{e}\end{array}$ & $\begin{array}{c}0,00000 \\
1\end{array}$ & $\begin{array}{c}1,00000 \\
0\end{array}$ & & $\begin{array}{c}\mathbf{0 , 3 5 2 7 0} \\
0\end{array}$ & $\begin{array}{c}1,00000 \\
0\end{array}$ \\
\hline $\begin{array}{c}\text { LM256 } \\
40\end{array}$ & $\begin{array}{c}(\text { SI9T } \times \text { SI19T } \\
)\end{array}$ & Sain & $\begin{array}{c}0,00000 \\
1\end{array}$ & $\begin{array}{c}0,00222 \\
1\end{array}$ & $\begin{array}{c}\mathbf{0 , 3 5 2 7 0} \\
0 \\
\end{array}$ & & $\begin{array}{c}1,00000 \\
0\end{array}$ \\
\hline $\begin{array}{c}\text { LM252 } \\
86 \\
\end{array}$ & Témoin & & $\begin{array}{c}0,00000 \\
1\end{array}$ & $\begin{array}{c}0,04417 \\
9 \\
\end{array}$ & $\begin{array}{c}1,00000 \\
0\end{array}$ & $\begin{array}{c}1,00000 \\
0\end{array}$ & \\
\hline
\end{tabular}

Les valeurs de $p$ du test en italique indiquent qu'il existe une différence significative entre la ligne et la colonne

Les valeurs de $p$ du test en gras indiquent qu'il n'existe pas de différence significative entre la ligne et la colonne

\section{Discussions}

Les résultats des Tests de KRUSKAL-WALLIS ont montré qu'il existe une différence significative au niveau du Nombre Total de Régimes (NTR), du Poids Total de Régimes (PTR) et du Poids Moyen de régimes (PMR) produits par les différentes descendances. La descendance témoin (LM25286) a produit plus de régimes et a eu un poids total de régimes plus important que les autres. Elle est suivie par les descendances saines LM25100 et LM25640, puis par les descendances malades LM25373 et LM 25635. La production de régimes de ces différentes descendances a varié au cours des années mais l'ordre de classement des descendances malades est conservé au cours de ces années (avant, pendant et après l'apparition des symptômes de la fusariose). La production des différentes descendances n'est certes pas identique mais le cycle de reproduction des différentes descendances n'a pas été affecté par l'apparition des symptômes de la fusariose. En effet, Les analyses semblent montrer qu'il existe préférablement un effet descendance (origine du croisement) sur la production des arbres même si ceux présentant les symptômes ont eu un NTR et un PTR faible avec un PMR élevé. L'observation des symptômes ayant été faite en 2014, c'est-à-dire 9 ans après la replantation sur une parcelle à forte densité de fusariose, NASH et SNYDER (1962) avaient montré à ce sujet, qu'en replantation la maladie apparait dès la première 
année. Or, la quasi inexistence de symptômes externes chez les descendances, après 9 ans de replantation, confirme les observations d'ALLOU et al., (2003), selon lesquelles lorsqu'un clone est sensible, le taux de fusariose externe est plus élevé que celui des symptômes internes. Ceci montre que le clone sensible n'a aucune défense contre la maladie. Par contre, l'inverse s'observe au niveau des clones tolérants avec une rareté des symptômes externes et une présence de symptômes internes faibles, ou élevés pour certains clones de même sensibilité. Il est donc prudent d'affirmer que les descendances présentant les symptômes de la fusariose ne sont pas malades mais ces symptômes observés sont plutôt les signes de l'expression de la tolérance de ces descendances à la fusariose. Aussi, les variations de l'expression des symptômes au niveau des différentes descendances pourraient résulter de l'interaction hôte-pathogène (plante et champignon), qui conduirait à une élaboration de certaines substances inhibitrices empêchant l'action de l'agent pathogène. La qualité et la quantité de ces substances synthétisées par la plante est en rapport avec la sensibilité de celles-ci vis à vis du champignon. C'est pourquoi, l'absence de symptômes externe a été observée chez les descendances saines (ALLOU et al, 2003). Cette analyse est en accord avec celle de HILLOCKS (1986) qui, travaillant sur le cotonnier, a fait remarquer, chez les tiges infectées par Fusarium oxysporum f.sp. vasinfectum, la présence d'occlusions vasculaires et d'accumulation importante d'aldéhydes terpéniques empêchent l'expression des symptômes de la fusariose. Les résultats des analyses statistiques descriptives ont montré aussi l'existence d'une variation annuelle dans le cycle de production du palmier à huile. Cette variation observée dans le cycle de production des descendances préjugées malades n'est pas le fait de la fusariose. Certains auteurs affirment que la production du palmier varie au cours des années en fonction de certains facteurs climatiques comme la pluviométrie. D'après JACQUEMARD (1995), le palmier à huile atteint son meilleur potentiel de production avec une pluviométrie minimale de 1800 $\mathrm{mm} / \mathrm{an}$ et bien répartie sur toute l'année. En effet, plus la saison sèche est marquée et plus elle affecte le rendement de la palmeraie. L'optimum pour la culture du palmier à huile se situe au delà de 2000 heures d'ensoleillement par an avec un rayonnement global moyen supérieur à $12 \mathrm{MJ} / \mathrm{m} 2 /$ jour. Tout ceci peut permettre de confirmer que l'apparition des symptômes de la fusariose n'a pas eu d'influence sur le cycle de production de régimes des descendances présentant les symptômes de la maladie. Le regroupement des descendances en groupes homogènes indiquent aussi que les descendances LM 25640 (saine) et LM 25373 (malade) ont donné le même poids total de régimes et les descendances LM 25635 (malade) et LM 25640 (saine) ont donné le même poids moyen de régimes en dépit des différences observées au niveau du nombre de régimes. Ce regroupement a révélé aussi que le 
poids moyen de régimes de la descendance LM 25373 (malade) qui est le plus important quelques soit l'année d'observation est le fruit de l'héritage des caractères des origines parentaux. Ce résultat confirme que les descendances qui ont produit moins de régimes, ont donné les régimes les plus lourds. Cela s'explique par l'origine parentale de cette descendance, prise en exemple, qui est un hybride de type (Deli Dura $\times$ Tenera). Selon GASCON et de BERCHOUX, (1964), l'origine Deli Dura, développée en Asie, produit un petit nombre de gros régimes alors que l'origine Tenera de la Mé, développée en Côte d'Ivoire, produit un grand nombre de petits régimes. Les descendances étant sur la même parcelle, disposées de manière aléatoire et dans les même conditions, l'apparition des symptômes de la maladie chez les descendances LM25373 et LM25635 ne peut être due qu'au seul milieu mais aussi à d'autres facteurs d'origine génétique. D'après TAQUET et al., (1984), i1 existerait des différences importantes de tolérance à la fusariose selon la combinaison génétique des descendances. En effet les descendances LM25373 et LM25100 bien qu'ayant les mêmes origines parentales ont présenté des états symptomatiques différents. Il en est de même pour les descendances LM25635 et LM25640 qui ont aussi la même origine parentale. Tous ces quartre descendances ont en commun un ancêtre (DA10D×DA3D) qui a une sensibilité neutre face à la fusariose et elles sont toutes issues de différentes combinaisons de croisements avec ce dernier comme grand-parent. Selon MEUNIER et al., (1979), la tolérance à la fusariose est un caractère polygénique essentiellement de mode additif or faire différents combinaisons de croisements, signifie utiliser différents gamètes donc différentes compositions allèliques. C'est cette différence de composition allèlique qui explique l'apparition des symptômes de la maladie chez les descendances LM25373 et LM25635. Ces descendances ont sans doute reçu plus d'allèles sensibles à la fusariose de leur ancêtre commun.

\section{Conclusion}

L'apparition des symptômes de la fusariose après 9 ans en replantation n'a eu aucun effet sur le nombre, le poids total et le poids moyen de régime produits par les descendances dites malades LM25373 et LM25635. La différence observée au niveau de la production et du nombre de régimes est due à l'origine génétique des descendances. La variation observée dans le cycle de production annuelle serait le fait de facteurs naturels tels que le changement climatique et la pluviométrie. Les semences qui seront produites à partir de la reproduction exacte des descendances LM25373 et LM25635 pourront être commercialisés car les caractères intéressants pour les producteurs ne sont pas affectés par la présence endémique à haute intensité de la fusariose vasculaire. 


\section{Conflit d'intérêt}

Les auteurs déclarent qu'ils n'ont aucun conflit d'intérêt.

\section{Contributions des auteurs}

Kouakou K O a réalisé la saisie des données, l'analyse statistique, la recherche bibliographique et à contribuer aux réflexions dans la discussion.

Diabaté $\mathrm{S}$, le chef du programme palmier à mise en place une équipe pour identifier les symptômes, prélever des échantillons pour confirmer la présence du champignon. N'guetta A S P, Directeur du laboratoire de génétique a coordonné et orienté ce travail. Il a donné un contenu scientifique basé sur ses connaissances dans le domaine de la génétique et amélioration des plantes. Allou D responsable de cet essai a initié la rédaction de l'article. Il a situé le contexte, identifier la problématique, défini l'objectif de cet travail. Il a suivi la collecte des données et apporté ces critiques pendant les différentes étapes de la rédaction. Il a aussi approuvé la méthodologie adoptée et participé à la rédaction et la correction du manuscrit.

\section{Remerciements}

Nos remerciements vont à l'endroit du Centre National de Recherche Agronomique qui a mis à notre disposition l'essai de la parcelle D9-3.

\section{References:}

1. ALlOU K., AHOUSSOU N., AKE S., DIABATE S., FRANQUEVILLE H (2003). Comportement de clones de palmier à huile au champ en zones de haute densité de Fusarium oxysporum $\mathrm{f}$. sp elaeidis en Côte d'Ivoire. Agron. Afr., 15(1): 29-38.

2. DAGNELIE P (1986). Analyse statistique à plusieurs variables. Gembloux, Presses agronomiques, $362 \mathrm{p}$.

3. DIABATE S (2009). La fusariose du palmier à huile : diagnostic et prévention ; fiche palmier $\mathrm{n}^{0} 2$, Ed. CNRA Avril 2009

4. DIABATE S., KONAN K J N., ALLOU D., HALA N'KLO F (2013). Effet d'une souche non pathogène de Fusarium oxysporum sur l'expression de la fusariose chez le palmier à huile (Elaeis guineensis Jacq.). Int. J. Biol. Chem. Sci. 7(3): 1279-1288, June 2013. Available online at http://ajol.info/index.php/ijbcs

5. DIABATE S., GBONGUE LR., DICK E., BOMISSO E L et FRANQUEVILLE H (2012). Etude Comparée de L'action de Souches non Pathogènes de Fusarium oxysporum dans l'induction des réactions immunitaires du palmier à huile contre la fusariose. European Journal of Scientific Research 3(2) : p. 193-201. 
6. DIABATE S., DEMBY K L., KOUASSI B G., WONGBE Yte, KONAN KJN., KONAN E., KOUABENAN A (2015). Etude de l'influence du facteur antécédent cultural palmiers et cocotiers sur l'évolution de la fusariose vasculaire chez six clones de palmiers à huile de Côte d'Ivoire. J. Appl. Biosci. Journal of Applied Biosciences 92: p $8570-8577$

7. DURAND-GASSELIN T., KOUAME KOUAME R., COCHARD B., ADON B., AMBLARD P (2000). Diffusion variétale du palmier à huile (Elaeis guineensis Jacq.), Oléagineux, Corps Gras, Lipides. Volume 7, Numéro 2, 207-14, Mars - Avril 2000, Dossier : Afrique, plantation et développement

8. FAO (2013). Bilans alimentaire: production mondiale de l'huile de palme. Division de la Statistique, FAOSTAT, Série informatique, FAO, Rome, Italie. Plantation, Recherche, Développement. Juilletaout. p5-10.

9. GASCON JP., DE BERCHOUX C., (1964). Caractéristiques de la production d'Elaeis guineensis (Jacq.) de diverses origines et de leurs croisements - Application à la sélection du palmier à huile.

Oléagineux 19(4): 75-84.

10. GOGBE D B F., KONAN J N., DIABATE S., KONAN E P., KONE B et DOGBO D O (2016). Réaction phénolique de quatre clones de palmier à huile inoculés par Fusarium oxysporum $f$. sp. Elaeidis, Int. J. Biol. Chem. Sci. 10(2): 486-496, April 2016, Available online at http://www.ifg-dg.org

11. HILLOCKS R J (1986). Cross protection between strains of Fusarium Oxysporum f. sp. vasinfectum and its effect on vascular resistance Mechanisms. Phytopathology : 117-216

12. HUBERT O (2010). Contribution de la production d'huile de palme au développement durable. Oléagineux, Corps gras, Lipides, 17(6) : 362- 367.

13. JACQUEMARD J C (1995). Le palmier à huile, Ed, Maisonneuve et Larose paris, $207 \mathrm{p}$

14. MEUNIER J M., RENARD J L., QUILLEC G (1979). Hérédité de la résistance à la fusariose chez le palmier à huile Elaeis guineensis Jacq. Application à la sélection du palmier à huile. Oléagineux, $\mathrm{n}^{0} 11$, p 661 .

15. NASH et SNYDER (1962), Quantitative estimation by plate counts of propagules of the been root rot Fusarium in field soil.

Physiopathology 52 : 567-572.

16. RENARD J L., NOIRET J M., MEUNIER J (1980). Source et gammes de résistance à la fusariose chez les palmiers à huile Elaeis 
guineensis et Elaeis melanococca, Oléagineux, vol. 35, n. 8-9, p.387-393 : 5 tabl., 1 ill., 3 réf.

17. RENARD J L., DE FRANQUEVILLE H (1989). Le palmier à huile, Oleagineux, vol. 44, n. 7, p. 341-349: 7 ill., 11 réf., 1 tabl. Conseil de l'IRHO n. 300.

18. ROYSTON P (1995). Aremark on algorithm AS 181: The W test for normality. Applied Statistics, 44, p 547-551.

19. SIEGEL S (1995). Non parametric statistic for the behavioral sciences. Mc Graw-Hill, New-York

20. SNEDECOR GW, COCHRAN WG. Statistical Methods, 7th ed. Ames: Iowa State University Press, 1980, p.476

21. TAQUET B, RAVISE A, Renard JL (1985). Modulation des réactions de défense du palmier à huile contre le FUSARIUM OXYSPORUM f. sp. ELAEIDIS (Schlecht) Toovey : applications : prémunition et stimulation chimique. Phytopathologische Zeitschrift, 1985, 112 (4), p. 298-314. ISSN 0031-9481 